\title{
Um software-protótipo sobre os direitos dos pacientes renais crônicos
}

\author{
A prototype software on the rights of chronic kidney patients \\ Un prototipo de software sobre los derechos de los pacientes renales crónicos
}

Recebido: 06/04/2021 | Revisado: 13/04/2021 | Aceito: 18/04/2021 | Publicado: 03/05/2021

\author{
Carolina do Val Alonso \\ ORCID: https://orcid.org/0000-0003-1431-285X \\ Universidade Federal do Estado do Rio de Janeiro, Brasil \\ E-mail: carolinadovalalonso@gmail.com \\ Lidiane da Fonseca Moura Louro \\ ORCID: https://orcid.org/0000-0003-3565-0567 \\ Universidade Federal Fluminense, Brasil \\ E-mail: lidimoura@outlook.com \\ Roberto Carlos Lyra da Silva \\ ORCID: https://orcid.org/0000-0001-9416-9525 \\ Universidade Federal do Estado do Rio de Janeiro, Brasil \\ E-mail: proflyra@gmail.com \\ Daniel Aragão Machado \\ ORCID: https://orcid.org/0000-0003-0680-5291 \\ Universidade Federal do Estado do Rio de Janeiro, Brasil \\ E-mail: daragao23@gmail.com \\ Yonara Cristiane Ribeiro \\ ORCID: https://orcid.org/0000-0002-6868-1629 \\ Universidade Federal Fluminense, Brasil \\ E-mail: yonara.cristiane@hotmail.com \\ Thiago Quinellato Louro \\ ORCID: https://orcid.org/0000-0001-8371-628X \\ Universidade Federal Fluminense, Brasil \\ E-mail: thiagolouro@hotmail.com
}

\begin{abstract}
Resumo
$\mathrm{O}$ acometimento de doenças crônicas tem ocorrido em grande escala, o estilo de vida relacionado com a genética dos indivíduos tem acarretado em maiores números, com isso o número de pacientes renais crônicos tem se tornado cada vez maior. Entende-se que o paciente renal crônico em tratamento dialítico tem a necessidade de saber seus direitos, o que pode acarretar em melhora na sua realidade social e de tratamento em saúde, e a existência de uma ferramenta tecnológica para suporte é de relevante existência. O estudo teve como objetivo: Criar um software protótipo acerca dos direitos dos pacientes renais crônicos em tratamento hemodialítico. Metodologia: Antes da realização da prototipação, foi realizada uma busca nas bases de dados, afim de descobrir a necessidade real da existência da criação do protótipo, para isso realizou-se o Scoping Review. O delineamento do estudo foi através do modelo de metodologia cíclica, de prototipagem. Resultados: $\mathrm{O}$ resultado foi apresentado ao longo de três etapas, que pontuam as etapas de construção de um protótipo. Através do desenvolvimento do estudo foi possível criar um software protótipo acerca dos direitos dos pacientes renais crônicos em tratamento hemodialítico. Conclusão: Conclui-se, portanto, que o objetivo do estudo foi concluído, visto a criação do protótipo. O protótipo tem grandes possibilidades de impacto na população alvo, visto que vem para somar como mais como mais uma ferramenta para manutenção dos direitos dos pacientes renais crônicos.
\end{abstract}

Palavras-chave: Doença renal; Tecnologia; Enfermagem.

\begin{abstract}
The involvement of chronic diseases has occurred on a large scale, the lifestyle related to the genetics of individuals has resulted in greater numbers, with this the number of chronic renal patients has become increasingly greater. It is understood that the chronic renal patient undergoing dialysis treatment needs to know his rights, which can lead to an improvement in his social reality and health treatment, and the existence of a technological tool for support is of relevant existence. The study aimed to: Create a prototype software about the rights of chronic kidney patients undergoing hemodialysis. Methodology: Before carrying out the prototyping, a search was carried out in the databases, in order to discover the real need for the existence of the creation of the prototype, for this purpose the Scoping Review was carried out. The study was designed using a model of cyclic methodology, of prototyping. Results: The result was presented over three stages, which punctuate the stages of building a prototype. Through the development of the study, it was possible to create a prototype software about the rights of chronic renal patients undergoing hemodialysis. Conclusion: It is concluded, therefore, that the objective of the study was concluded,
\end{abstract}


considering the creation of the prototype. The prototype has great possibilities of impact on the target population, since it comes to add more as another tool for maintaining the rights of chronic kidney patients.

Keywords: Kidney disease; Technology; Nursing.

\section{Resumen}

La implicación de enfermedades crónicas se ha producido a gran escala, el estilo de vida relacionado con la genética de los individuos ha resultado en mayor número, con ello el número de pacientes renales crónicos se ha vuelto cada vez mayor. Se entiende que el paciente renal crónico en tratamiento de diálisis necesita conocer sus derechos, lo que puede derivar en una mejora en su realidad social y tratamiento de salud, y es de relevancia la existencia de una herramienta tecnológica de apoyo. El estudio tuvo como objetivo: Crear un prototipo de software sobre los derechos de los pacientes renales crónicos en hemodiálisis. Metodología: Antes de realizar el prototipo, se realizó una búsqueda en las bases de datos, con el fin de descubrir la necesidad real de la existencia de la creación del prototipo, para ello se realizó la Scoping Review. El estudio se diseñó utilizando un modelo de metodología cíclica, de prototipado. Resultados: El resultado se presentó en tres etapas, que marcan las etapas de construcción de un prototipo. A través del desarrollo del estudio, fue posible crear un prototipo de software sobre los derechos de los pacientes renales crónicos en hemodiálisis. Conclusión: Se concluye, por tanto, que se concluyó el objetivo del estudio, considerando la creación del prototipo. El prototipo tiene grandes posibilidades de impacto en la población objetivo, ya que viene a sumar más como una herramienta más para mantener los derechos de los pacientes renales crónicos.

Palabras clave: Enfermedad renal; Tecnología; Enfermería.

\section{Introdução}

O acometimento de doenças crônicas tem ocorrido em grande escala no Brasil, relacionada com diversos fatores, dentre esses o estilo de vida, assim como a genética das pessoas, o que tem acarretado com o crescimento do número de casos de indivíduos acometidos por lesão renal crônica. A patologia tem como causas principais a idade, o sexo, a diabetes, a hipertensão arterial sistêmica, a proteinúria, a anemia, as complicações metabólicas, a obesidade, o tabagismo e a dislipidemia (Bastos \& Kirsztajn, 2011). Assim, a referida doença se configura em uma constante preocupação para o Brasil, assim como para o mundo.

Como consequência do constante aumento no número de casos de indivíduos acometidos pela doença renal crônica, deu-se o aumento dos indicadores relacionados ao tratamento. Assim em 2014, a taxa de prevalência de tratamento de dialise foi de 552 pacientes por milhão de pessoas, a taxa de prevalência mundial teve aumento dos anos de 2011 a 2013 de 6\% (Sesso et al, 2016).

Em meio a todo esse contexto atual, cabe inserir o emergir da tecnologia como aliada para diminuição de impactos na saúde e assim como para o desenvolvimento do cuidado e tratamento. Dessa maneira, o uso cada vez maior de ferramentas tecnológicas, se tornaram grandes aliadas para pacientes e profissionais quando o assunto é suporte terapêutico ao tratamento, de maneira educativa, extrapolando assim a figura do aparelho de hemodiálise, utilizado diariamente neste tipo de tratamento.

A tecnologia e a inovação tecnológica são citadas nos meios de comunicação, agendas governamentais, empresas, agências de fomento de pesquisas e inúmeras organizações sociais como grande influência na área da saúde. Lorenzetti et al (2012, p.433) citam que: "ciência e tecnologia são instrumentos importantes para a saúde e o tratamento de doenças, assim como para a construção de um momento civilizatório de paz e de vida digna e decente para todos".

Arone e Cunha (2007) ressaltaram que os avanços científicos e tecnológicos ocorridos no último século, causaram impactos diretos em organizações e profissionais de saúde, neste interim também está inserida a enfermagem.

Para Silva e Louro (2010) a enfermagem como ciência e área pertencente a saúde, utilizou-se dos meios de tecnologia para aprimoramento de seu trabalho, utilizando a tecnologia atualmente como ferramenta para proporcionar uma melhora no assistir junto ao seu paciente e como facilitadora de seu cuidado. Com a fundamentação cientifica do cuidado de enfermagem ocorreu o reconhecimento da sentença tecnológica do cuidado, reconhecido como processo e produto. Afinal, a tecnologia e o cuidado estão ligados com a história da civilização humana.

Entende-se que o paciente renal crônico em tratamento dialítico tem a necessidade de saber seus direitos, o que pode 
acarretar em melhora na sua realidade social e de tratamento em saúde, e a existência de uma ferramenta tecnológica para suporte é de relevante existência. Frente essa realidade o presente estudo teve como objeto: os direitos dos pacientes renais crônicos em tratamento dialítico.

Perante as afirmativas anteriormente expostas o presente estudo se justificou tendo em vista o embasamento legal a partir da Lei de $n^{\circ} 12.527$ (Brasil, 2011) e do Decreto de $n^{\circ} 7.724$ (Brasil, 2011), que consideraram a relevância dos pacientes crônicos terem informações sobre suas patologias e ainda podendo ter acesso ao conhecimento de seus direitos pessoais, afim de garanti-los.

Neste interim, podemos ainda mencionar a existência da Lei de $\mathrm{n}^{\circ} 7.853 / 89$, que trata do suporte às pessoas portadoras de deficiências, mencionando que a garantia do exercício de seus direitos pessoais e sociais, possibilitam o desenvolvimento de sua integração social (Brasil, 1989).

Como questão norteadora tivemos: Quais são as etapas para a criação de um software protótipo acerca dos direitos dos pacientes renais crônicos em hemodiálise?

Como objetivo foi traçado: Criar um software protótipo acerca dos direitos dos pacientes renais crônicos em tratamento hemodialitico.

\section{Metodologia}

Tratou-se de uma pesquisa qualitativa, sendo um estudo metodológico, desenvolvido através da Prototipação. A coleta e análise dos dados de pesquisas qualitativas demandam grande envolvimento do pesquisador, devido sua variedade de dados narrativos (Handem, 2009).

Através do estudo foi possível criar um software-protótipo, viável para execução em celulares e tablets, mais precisamente na forma de aplicativo, assim como modelo proposto de protótipo por Presman (2011).

O delineamento do estudo foi através do modelo de metodologia cíclica, de prototipagem, Presman (2011) cita que para construção de um protótipo é necessário que sejam realizadas as etapas de coleta e refinamento de requisitos, o projeto rápido, a construção do protótipo, a avaliação do protótipo, o refinamento do protótipo e a engenharia do projeto.

Em todo momento foram respeitadas as normas descritas pela Resolução do Conselho Nacional de Saúde de $\mathrm{n}^{\circ} 510$ de 2016 (Brasil, 2016), que trata do uso de dados de domínio público.

Presman (2011) traz que o processo de prototipagem conta com quatro etapas básicas, das quais são aplicadas cinco atividades metodológicas para a criação de aplicativos para internet. A primeira etapa é da comunicação, a segunda conta com o planejamento rápido, a terceira etapa com a construção em si do protótipo e a quarta com o emprego do produto, e seus testes. O estudo realizou só as três primeiras etapas.

Quanto a realização da criação teve-se como base o estudo desenvolvido por Louro (2019), que criou um aplicativo baseado nos mesmo conceitos e plataforma de criação.

Inicialmente como proposto por Pressman (2011), estabeleceu-se o objetivo do protótipo, ou seja, a primeira etapa ou fase de comunicação, discorrendo sobre o objetivo do protótipo. A fase ainda é subdividida em três outras etapas: identificação das necessidades de informação dos pacientes renais crônicos. A observação e mapeamento da existência de tecnologias para os pacientes renais crônicos, nas bases de aplicativos. A identificação dos dispositivos tecnológicos existentes, que fossem de fácil acesso e de simples compreensão para os pacientes renais crônicos.

Iniciou-se o planejamento do software, que é a segunda etapa descrita por Pressman (2011), onde definiu-se inicialmente qual seria o sistema operacional a ser utilizado, realizou-se uma previsão orçamentária, definiu-se os recursos visuais, gráficos e a escrita, para isso o software foi desenvolvido para seu uso em Android, IOS e navegadores de internet.

$\mathrm{Na}$ última etapa do estudo, classificada com a terceira etapa descrita por Pressman (2011), discorrendo sobre a 
construção do protótipo, por um profissional de tecnologia da informação. Para isso, o protótipo foi desenvolvido no site "Fábrica de aplicativos - Fabapp" (link: https://fabricadeaplicativos.com.br/), através da tecnologia Progressive Web App, que é aplicada pelo Google Inc., para sua utilização em meios digitais.

É necessário apenas compartilhar o endereço do protótipo, que pode ser acessado por qualquer pessoa para acesso ao conteúdo do aplicativo.

\section{Resultados}

\section{$1^{\circ}$ Etapa: Comunicação - Identificação das tecnologias disponíveis sobre tecnologia e nefropatias.}

É a etapa mais importante da construção do protótipo, trata da relevância da construção do protótipo sobre o tema definido e estabelece formas de ampliação e para melhoria de acesso aos dispositivos de softwares.

Foram identificadas as necessidades da criação do software em um estudo prévio, a partir de um levantamento acerca da existência de tecnologias (aplicativos) voltados para pacientes renais crônicos em tratamento hemodialitico com informações referentes aos seus direitos e tratamento.

A seguir foi realizado um levantamento sobre a existência de softwares (aplicativos) na vertente do estudo. Através dos resultados os dados serão apresentados através do: Custeio, idioma, público alvo e finalidade. Os dados foram identificados nas plataformas de lojas do App Store (IOS) e Play store (Android).

Onde pesquisou-se no no App store, aplicativo do IOS, por "tecnologia nefropatias", e se localizou 0 resultados, ainda que o foco do estudo seja pesquisar por tecnologias relacionadas a nefropatias, pesquisou-se pelo termo "nefropatias", e nada foi evidenciado, contando com 0 resultados no App Store.

No Play Store, aplicativo Android, evidenciou-se 10 resultados para "tecnologia nefropatias". Já para "nefropatias", encontrou-se 10 resultados, mas o termo foi utilizado apenas visando encontrar algo na plataforma IOS, com isso foram considerados apenas os resultados dos aplicativos sobre "tecnologia nefropatias". Dos quais 06 apenas tratam do assunto, os outros 04 eram sobre antivírus (02), aplicativo de passageiro e monitor cardíaco.

Com a pretensão de desenvolver conhecimento para o Brasil, procurou-se por aplicativos que tivessem com o idioma principal o país (português), dos 06 aplicativos encontrados apenas 01 foi desenvolvido na língua do país, os outros cinco foram desenvolvidos em inglês.

Figura 1 - Estratificação dos aplicativos segundo o idioma.

\begin{tabular}{|c|c|}
\hline Idioma & Play store \\
\hline Português & $16,66 \%(01)$ \\
\hline Outros idiomas & $83,33 \%(05)$ \\
\hline Total: & $100 \%(06)$ \\
\hline
\end{tabular}

Fonte: Autores.

Evidenciou-se na Figura a predominância de aplicativos em inglês, conclui-se então, a necessidade do desenvolvimento de aplicativos em português, pois existem também profissionais e pacientes nefrológicos no país. Visto o software desenvolvido ser focado para as necessidades brasileiras, focou-se o resultado no aplicativo que possui dados em português:

É um aplicativo voltado para nefrologia, então $100 \%$ dos aplicativos estão relacionados a nefrologia, é uma calculadora na qual se opta por 05 fórmulas para que cálculo da taxa de filtração glomerular estimada. Não se identificou o 
local de origem do aplicativo, mas é um aplicativo da REDA BRAHIMI. Não é um aplicativo totalmente gratuito, a versão plus da calculadora é paga.

Tem por finalidade ser uma calculadora para profissionais da saúde calcularem as medidas. Nenhum dos aplicativos tratava dos direitos dos pacientes renais crônicos em tratamento hemodialítico. Sendo essencial a criação de um aplicativo para o público.

\section{Etapa 2 - Planejamento Rápido}

Nessa etapa é realizado um rascunho das funções do software, é realizada uma representação sistemática do que comporá o software, contendo os requisitos básicos de realização de um projeto, com seu sistema operacional, apresentação do aplicativo, orçamento, ícones contidos no aplicativo.

Pressman (2011) trata que a etapa deve conter um conjunto de práticas técnicas e gerenciais, que vão direcionar, como um roteiro, para as ações traçadas com os objetivos propostos, citando que a modelagem deve conter as funções, arquitetura e informações que serão utilizadas para construção do software, ou seja as informações que o sistema terá. As etapas para construção são:

$1^{\circ}$ passo: definição de sistema operacional de uso;

$2^{\circ}$ passo: planejamento orçamentário;

$3^{\circ}$ passo: definição de esquema gráfico;

$4^{\circ}$ passo: definição de conteúdo escrito.

$1^{\circ}$ Passo: Definição de sistema operacional de uso

Quando o assunto é a disponibilização de conteúdos por meio de mídias digitais, mais precisamente aplicativos, têmse duas grandes plataformas de aplicativos, que geram conteúdos, o APP Story e o Play Story, disponibilizados pelo IOS e Android. O sistema operacional foi voltado para smartphones, devido ao grande uso da tecnologia na contemporaneidade.

$2^{\circ}$ Passo: Planejamento Orçamentário

Visto a definição da plataforma utilizada, avaliou-se os custos para implementação do software protótipo. Para isso foi utilizado o site (www.quantocustaumapp.com.br), da Aioria.

Assim, para definir o orçamento, o contratante responde um questionário, com informações pré-definidas, e é apresentada a cotação para criação do aplicativo. O projeto é enviado aos profissionais da empresa para que seja desenvolvido. A cotação obtida foi de $\mathrm{R} \$ 64.000,00$ (sessenta e quatro mil reais), cabe mencionar que a pesquisa não possuiu financiamento, ou ajuda orçamentária.

$3^{\circ}$ Passo: Definição de esquema Gráfico:

Para elaboração do software, realizou-se um esquema gráfico, que viabilizou a compreensão e visualização do protótipo. A seguir será apresentado: 
Figura 2 - Esquema gráfico do software-protótipo.

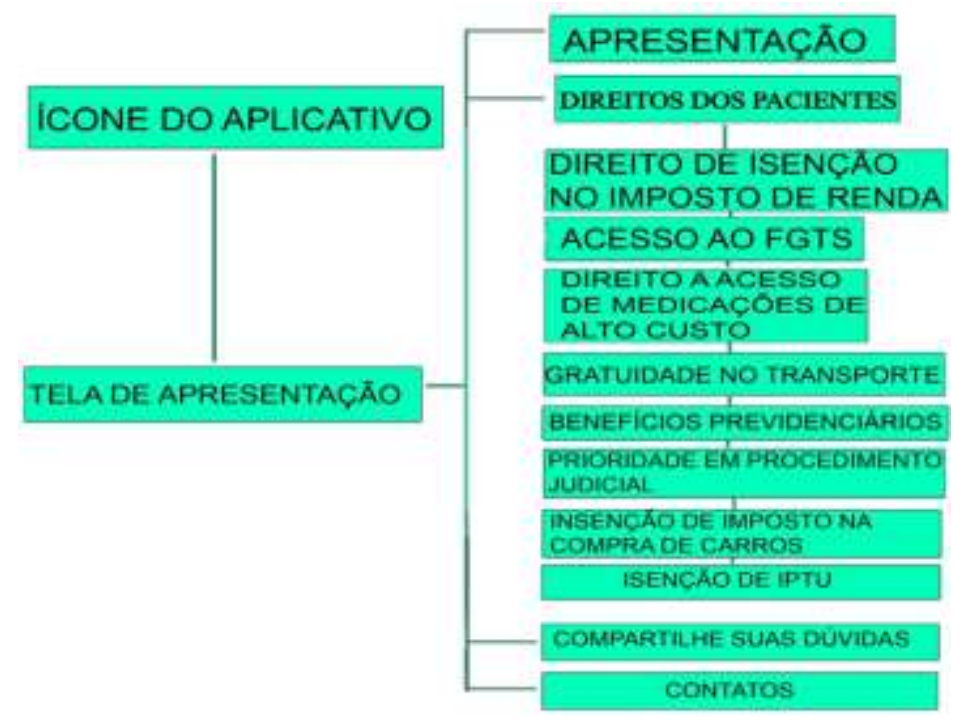

Fonte: Autores.

Como visto na figura 06, o acesso ao software será da seguinte forma: A página será acessada através da plataforma Fabapp, no link: https://app.vc/nefro_direitos, o usuário encontrará o software por meio de seu ícone, o site irá direcioná-lo para baixar o aplicativo de forma gratuita, e instalará no seu dispositivo, o Fabapp disponibiliza leito de QR Code, para que o usuário acesse o Nefro Direitos, como pode se observar na figura a seguir:

Figura 3 - Template de Acesso.

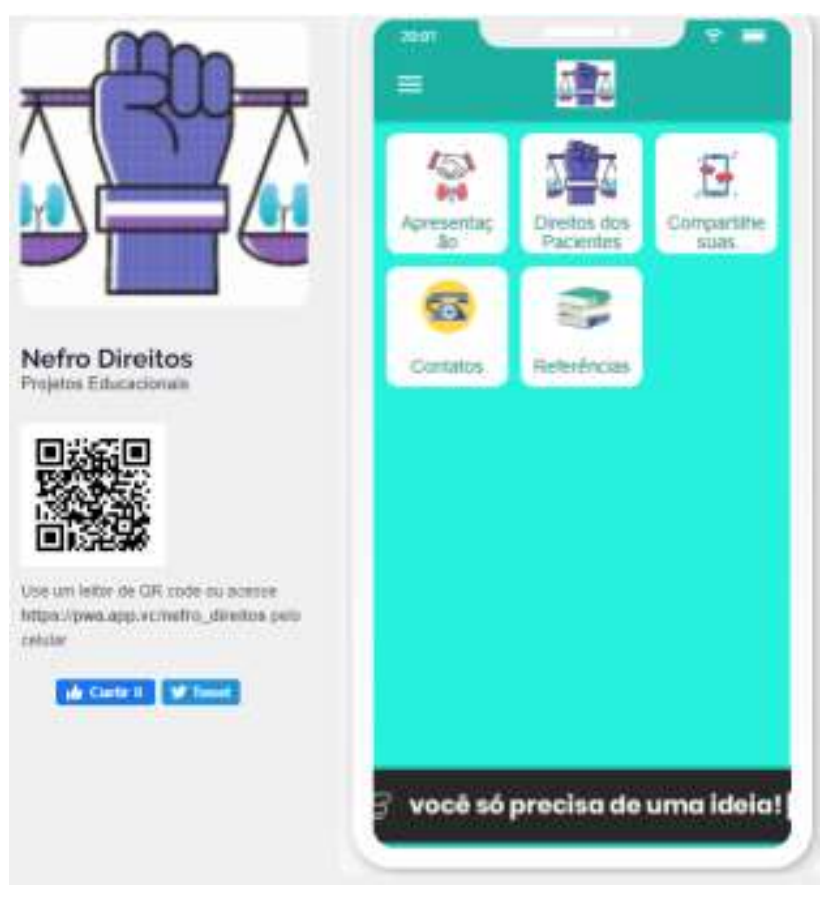

Fonte: https://app.vc/nefro_direitos.

Ao instalar o aplicativo, o usuário será redirecionado ao Menu principal, onde encontrará as abas de menu, que contemplam a apresentação, os direitos, compartilhe suas dúvidas, contato e referências. 
Quando clicar nas abas dos direitos, será direcionado a aba que contém a descrição, com apoio legal de seus direitos, como na Figura 3.

$4^{\circ}$ Passo: Definição do conteúdo escrito:

Para corresponder ao objetivo do estudo, foram levantados os materiais escritos para o aplicativo. Levantou-se as necessidades e se realizou-se buscas em documentos. O aplicativo tem o objetivo de apresentar alguns dos direitos dos pacientes renais crônicos, é interessante citar que mesmo com a legislação vigente por vezes se faz necessário a utilização de medidas legais e interpretação para sua utilização e garantia. O conteúdo escrito que tange sobre os direitos dos pacientes renais crônicos foi descrito da seguinte forma:

- Isenção no Imposto de Renda: O paciente Renal Crônico tem direito a isenção no imposto de renda, a Lei $n^{\circ} 7.713$ (Brasil,1988), em seu artigo $6^{\circ}$ estabelece que são isentos do imposto de renda de pessoa física os portadores de nefropatia grave.

- Acesso ao FGTS: segundo a Lei no 8.036 (Brasil,1990) os portadores de doenças graves e pacientes em estágio de doença terminal tem direito de utilizar o FGTS.

- Direito a acesso de medicações de alto custo: segundo a Lei no 8.080 (Brasil,1990), a saúde é direito do cidadão e deve ser assegurada pelo estado, que deve propiciar condições para isso, frente a essa afirmativa cabe ao estado a garantia de provento das medicações de alto custo, como as de pacientes renais crônicos.

- Gratuidade no transporte: segundo a Lei de $n^{\circ} 8.899$ (Brasil,1994) e decreto $n^{\circ} 3.691$ (Brasil, 2000), o paciente renal crônico tem direito a transporte gratuito, que é concedido a pessoas com deficiência e comprovada carência.

- Benefícios previdenciários: aposentadoria por invalidez e auxílio doença em casos específicos, segundo a Lei complementar no 142 (Brasil, 2013) e Decreto nº 3.048 (Brasil, 1999).

- Prioridade em procedimento judicial: segundo a Lei $n^{\circ} 12.008$ (Brasil, 2009), que menciona que o portador de doença grave tem direito a prioridade.

- Isenção de imposto na compra de carros: segundo a Lei no 8.989 (Brasil,1995) a pessoa portadora de deficiência está isenta de impostos o sobre produtos industrializados.

- Cabe destacar que alguns municípios dispõem sobre a isenção de IPTU, sendo necessário se consultar o da cidade do portador de doença renal crônica.

\section{Etapa 3 - Construção do Protótipo}

A última etapa contemplada pelo estudo, como descrito anteriormente, pois a validação será futuramente objeto de outro estudo, é a etapa em que serão apresentadas as partes visuais do software, como está disponível para os usuários. Nomeado de nefro direitos, visa informar e possibilitar conteúdo legal e digital para os pacientes renais crônicos, acerca de seus direitos.

O menu principal compreende de forma clara e didática: Menu inicial; Apresentação; Direitos dos Pacientes (Direito de Isenção no Imposto de Renda, Direito de acesso ao FGTS, Direito Medicação Alto Custo, Gratuidade no Transporte, Benefícios Previdenciários, Prioridade de Justiça, Isenção Compra de Carro, Isenção IPTU) Compartilhe suas Dúvidas; Contato; Referências.

A figura a seguir trata do Menu suspenso e do Menu inicial: 
Figura 4 - Menu Suspenso e Inicial.

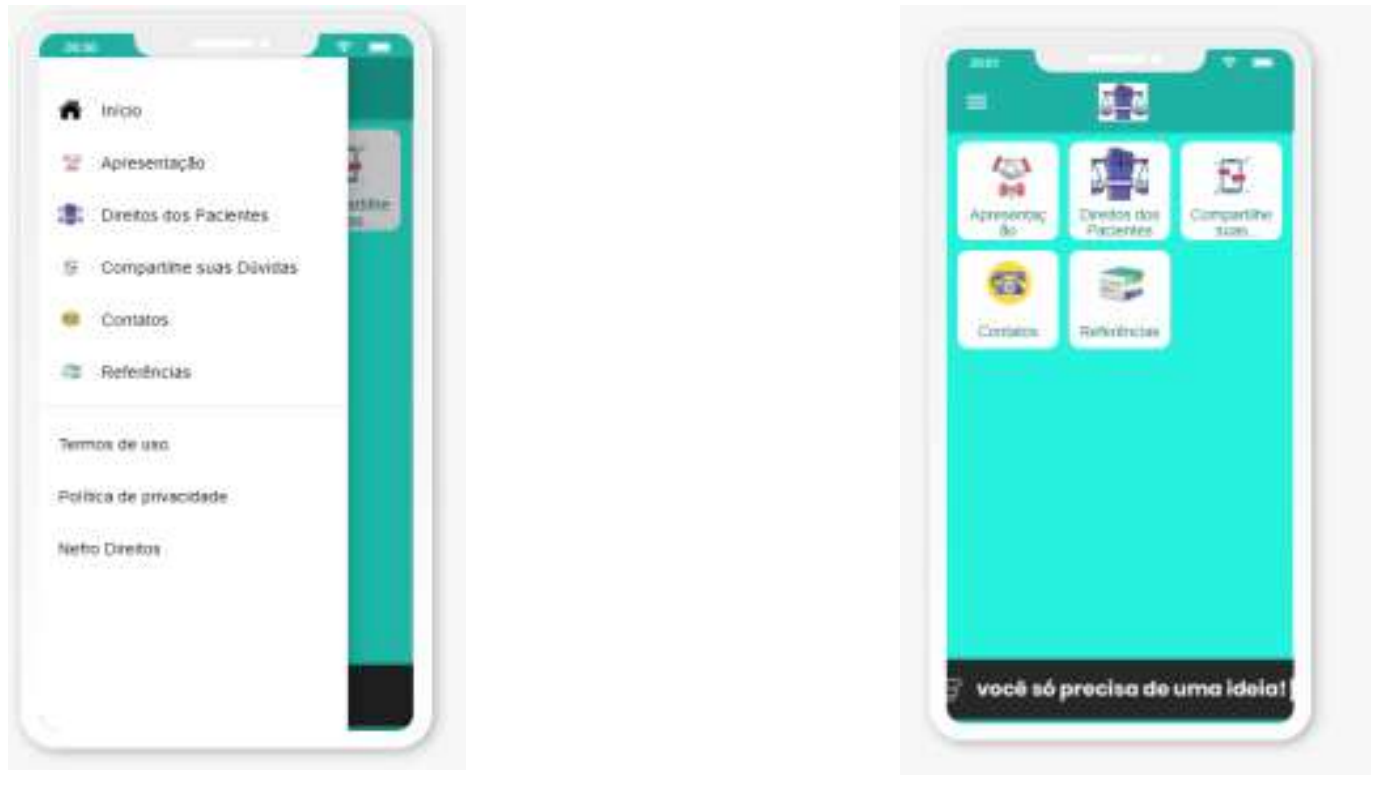

Fonte: https://app.vc/nefro_direitos.

\section{Discussão}

Os direitos dos pacientes renais crônicos são de grande importância em sua vida, pois podem diminuir impactos na sua vida pessoal, familiar e laboral. O paciente renal através de seus direitos pode assegurar uma melhora em sua qualidade de vida, o que pode impactar até é uma melhora em sua qualidade de vida. Chaves, Costa, Lunardi (2005, p.38) afirmam que "Todo paciente tem direito a ser reconhecido e respeitado como cidadão, o que implica participar das decisões relacionadas ao seu cuidado e tratamento".

Como apontado nos resultados a Lei $n^{\circ} 7.713$ (Brasil,1988), cita seu artigo $6^{\circ}$ que são isentos do imposto de renda de pessoa física os portadores de nefropatia grave. O que é de grande relevância, visto que por conta do tratamento muitos pacientes por vezes comprometem sua renda, pois necessitam de uma alimentação regrada, dentre outras necessidades, o que proporciona um gasto maior, e a isenção do imposto de renda passa a ser uma economia para aos pacientes que precisam declarar.

O Acesso ao FGTS é de grande relevância quando o assunto é o aumento de gastos, a Lei no 8.036 (Brasil,1990) assegura que os portadores de doenças graves e pacientes em estágio de doença terminal podem e tem o direito de utilizar o seu FGTS.

Assim como apontado nos resultados a Lei no 8.080 (Brasil,1990), define que a saúde é direito do cidadão e deve ser assegurada pelo estado, que deve propiciar condições para isso, como isso cabe ao estado a garantia de provento das medicações de alto custo, como as de pacientes renais crônicos. Assegurar o acesso a medicação de alto custo é essencial para manutenção do tratamento de pacientes de doenças crônicas, no caso do paciente renal crônico, em que as medicações são essenciais para o tratamento.

A Gratuidade no transporte que é assegurada pela Lei de no 8.899 (Brasil,1994) e pelo decreto no 3.691 (Brasil, 2000), é primordial para realização do tratamento nefrológico, visto que esses pacientes precisam estar na clínica de dialise várias vezes por semana, e muitos dependem de transporte público, e não possuem recurso, com o direito, asseguram sua locomoção para as clinicas de diálise. 
Como todo trabalhador os pacientes renais crônicos possuem seus Benefícios previdenciários, tais como a aposentadoria por invalidez, o auxílio doença em casos específicos, e segundo a Lei complementar $\mathrm{n}^{\circ} 142$ (Brasil, 2013) e Decreto nº 3.048 (Brasil, 1999), ficam resguardados.

A Prioridade em procedimento judicial que é garantida pela Lei $\mathrm{n}^{\circ} 12.008$ (Brasil, 2009), que menciona que o portador de doença grave tem direito a prioridade traz respaldo até mesmo para garantia da validade dos direitos dos pacientes, visto que se necessário utilizarem de meios legais para fazerem valer seus direitos, terão uma maior rapidez em seu processo.

A compra de bens ainda é o sonho de muitos brasileiros, e a seguridade da Isenção de imposto na compra de carros, facilita a vida do portador de doença renal crônica, visto que na possibilidade de comprar um carro, pode estar isento do tributo, sabe-se da necessidade da presença do cliente na clínica de diálise e a compra de um carro para locomoção facilita sua locomoção, segundo a Lei no 8.989 (Brasil,1995) a pessoa portadora de deficiência está isenta de impostos o sobre produtos industrializados.

Quando o assunto é o IPTU, é necessário realizar uma verificação junto a Prefeitura da cidade de moradia, visto que os Munícipios podem dispor ou não da isenção. Sendo necessário se informar, mas alguns municípios já isentam. Mediante o observado se faz possível evidenciar a importância dos direitos dos portadores de doença renal crônica em tratamento dialítico.

\section{Conclusão}

Conclui-se, portanto, que o objetivo do estudo foi concluído, visto a criação do protótipo foi apresentada. A tecnologia desenvolvida no presente estudo tem grandes possibilidades de impacto na população alvo, visto que vem para somar como mais uma ferramenta para manutenção dos direitos dos pacientes renais crônicos, visto que tem se discutido constantemente a retirada de direitos por parte do governo frente a pessoa com deficiência, assim como noticiado uma possível retirada da isenção na compra de carros acima de valor estabelecido pelo atual governo.

Os direitos dos pacientes renais crônicos parecem escassos, mas são de extrema relevância para essa população e significam grande conquista. Dessa maneira pretendemos que a partir da oportunidade de desenvolvimento do presente estudo seja um marco inicial para uma ampliação nos debates não somente em nível acadêmico, mas também nas unidades assistenciais em saúde sobre os direitos pessoais dos indivíduos acometidos por doença renal crônica, uma vez que estes têm grande repercussão no cotidiano dessas pessoas.

A partir da finalização do presente estudo pensamos ser oportuno o desenvolvimento de outros estudos, quais sejam de validação do protótipo, a usabilidade do mesmo, e tantos outros vislumbrando a melhoria da qualidade final da tecnologia desenvolvida. Neste interim, cabe nesta oportunidade ressaltar a importância da realização de outros estudos, ainda no contexto da assistência de enfermagem aos indivíduos com doença renal crônica tendo em vista as necessidades de cuidados que podem emergir dessa clientela.

\section{Referências}

Arone, E. M.; \& Cunha, I. C. K. O. (2007). Tecnologia e humanização: desafios gerenciados pelo enfermeiro em prol da integralidade da assistência. Revista Brasileira de Enfermagem, 60(6), 721-723. https://dx.doi.org/10.1590/S0034-71672007000600019.

Bastos, M. G., \& Kirsztajn, G. M. (2011). Doença renal crônica: importância do diagnóstico precoce, encaminhamento imediato e abordagem interdisciplinar estruturada para melhora do desfecho em pacientes ainda não submetidos à diálise. Brazilian Journal of Nephrology, 33(1), 93-108. https://doi.org/10.1590/S0101-28002011000100013.

Brasil. (1988). Lei 7.713, de 22 de dezembro de 1988. Altera a legislação do imposto de renda e dá outras providências.

Brasil. (1989). Lei 7.853, de 24 de outubro de 1989. Dispõe sobre o apoio às pessoas portadoras de deficiência, sua integração social, sobre a Coordenadoria Nacional para Integração da Pessoa Portadora de Deficiência - Corde, institui a tutela jurisdicional de interesses coletivos ou difusos dessas pessoas, disciplina a atuação do Ministério Público, define crimes, e dá outras providências.

Brasil. (1990). Lei 8.036, de 11 de maio de 1990. Dispõe sobre o Fundo de Garantia do Tempo de Serviço, e dá outras providências. 
Brasil. (1990). Lei n. 8.080, de 19 de setembro de 1990. Dispõe sobre as condições para a promoção, proteção e recuperação da saúde, a organização e o funcionamento dos serviços correspondentes, e dá outras providências.

Brasil. (1994). Lei 8.899, de 29 de junho de 1994. Concede passe livre às pessoas portadoras de deficiência no sistema de transporte coletivo interestadual.

Brasil. (1995). Lei 8.989, de 24 de fevereiro de 1995. Dispõe sobre a Isenção do Imposto sobre Produtos Industrializados - IPI, na aquisição de automóveis para utilização no transporte autônomo de passageiros, bem como por pessoas portadoras de deficiência física, e dá outras providências.

Brasil. (1999). Decreto 3.048, de 06 de maio de 1999. Aprova o Regulamento da Previdência Social, e dá outras providências.

Brasil. (2000). Decreto 3.691, de 19 de dezembro de 2000. Regulamenta a Lei no 8.899, de 29 de junho de 1994, que dispõe sobre o transporte de pessoas portadoras de deficiência no sistema de transporte coletivo interestadual.

Brasil. (2009). Lei 12.008, de 29 de julho de 2009. Altera os arts. 1.211-A, 1.211-B e 1.211-C da Lei no 5.869, de 11 de janeiro de 1973 - Código de Processo Civil, e acrescenta o art. 69-A à Lei no 9.784, de 29 de janeiro de 1999, que regula o processo administrativo no âmbito da administração pública federal, a fim de estender a prioridade na tramitação de procedimentos judiciais e administrativos às pessoas que especifica.

Brasil. (2011). Lei 12.527, de 18 de novembro de 2011. Regula o acesso a informações previsto no inciso XXXIII do art. $5^{\circ}$, no inciso II do $\S 3^{\circ}$ do art. 37 e no $\S 2^{\circ}$ do art. 216 da Constituição Federal; altera a Lei ${ }^{\circ}$ 8.112, de 11 de dezembro de 1990; revoga a Lei ${ }^{\circ} 11.111$, de 5 de maio de 2005 , e dispositivos da Lei n ${ }^{\circ} 8.159$, de 8 de janeiro de 1991 ; e dá outras providências.

Brasil. (2011). Lei 7.724, de 18 de novembro de 2011. Regulamenta a Lei $\mathrm{n}^{\circ}$ 12.527, de 18 de novembro de 2011, que dispõe sobre o acesso a informações previsto no inciso XXXIII do caput do art. $5^{\circ}$, no inciso II do $\S 3^{\circ}$ do art. 37 e no $\S 2^{\circ}$ do art. 216 da Constituição.

Brasil. (2013). Lei Complementar 142, de 08 de maio de 2013. Regulamenta o § 1o do art. 201 da Constituição Federal, no tocante à aposentadoria da pessoa com deficiência segurada do Regime Geral de Previdência Social - RGPS.

Brasil. (2016). Conselho Nacional de Saúde. Resolução 510.

Chaves, P. L., Costa, V. T., \& Lunardi, V. L. (2005). A enfermagem frente aos direitos de pacientes hospitalizados. Texto \& Contexto - Enfermagem, 14(1), 38-43. https://doi.org/10.1590/S0104-07072005000100005.

Handem, P. C. et al. (2008). Método e metodologia na pesquisa científica: Metodologia: interpretando autores. (3a ed.), In: Fiqueiredo, N. M. A. Yendis Editora.

Lorenzetti, J. et al. (2012). Tecnologia, inovação tecnológica e saúde: uma reflexão necessária. Texto \& Contexto - Enfermagem, 21(2), 432-439. https://doi.org/10.1590/S0104-07072012000200023.

LOURO, L. F. M. (2019) Cuidados de Enfermagem a Pacientes submetidos a Quimioterapia Antineoplásica Ambulatorial: A criação de um SoftwareProtótipo. Tese de Doutorado, Universidade Federal do Estado do Rio de Janeiro, Rio de Janeiro RJ, Brasil.

Presman, R. S. (2011). Engenharia de software. (3a ed.), Pearson Makron Books.

Sesso, R. C. et al. (2016). Inquérito Brasileiro de Diálise Crônica 2014. Brazilian Journal of Nephrology, 38(1), 54-61. https://doi.org/10.5935/01012800.20160009

Silva, R. C. L., \& Louro, T. Q. (2010). The incorporation of the hard technologies in the care of nursing in intensive therapy and the development of the speech of the humanization. Revista de Enfermagem UFPE on line, 4(3), 1557-1564. https://doi.org/10.5205/reuol.1070-8483-2-LE.0403201030. 